\title{
BEAM COMMISSIONING OF A MULTI-PURPOSE COMPACT ION SYNCHROTRON
}

\author{
K. Matsuda, K. Saito, H. Nishiuchi, M. Umezawa, K. Hiramoto, R. Shinagawa, T. Tonooka \\ Hitachi, Ltd. Power \& Industrial Systems Group \\ 7-2-1, Omika-cho, Hitachi-shi, Ibaraki-ken, 319-1221 Japan
}

\begin{abstract}
W-MAST (Wakasa-wan Energy Research Center Multipurpose Accelerator with Synchrotron and Tandem) consists of a tandem accelerator with 5MV terminal voltage and a compact ion synchrotron. The beam commissioning was started at the beginning of 1999 and was successfully accomplished in March 2000. A proton beam of $10 \mathrm{MeV}$ was injected from the tandem accelerator and accelerated by the ion synchrotron to $200 \mathrm{MeV}$ during $0.6 \mathrm{~s}$. In order to reduce the space charge induced tune spread, the second order harmonic component was superposed onto the accelerating radio frequency field with an untuned accelerating cavity having FINEMET cores. Betatron tune during the acceleration was measured and controlled to improve the acceleration efficiency.
\end{abstract}

\section{INTRODUCTION}

W-MAST is an accelerator complex which was constructed in Fukui Prefecture, Japan. W-MAST consists of a $5 \mathrm{MV}$ tandem type electrostatic accelerator with two negative ion sources, an ion synchrotron and a $200 \mathrm{kV}$ micro-wave ion source [1-2]. Configuration of the accelerator complex is shown in figure 1. Many species of ion beams with a wide energy range are available in four irradiation rooms. Irradiation room 1 utilises a low-energy beam (lower than $3.4 \mathrm{MeV}$ for protons) from the tandem accelerator. Two beam lines are dedicated to element analysis, crystal structure analysis with the micro-PIXE course, and material science. A $200 \mathrm{kV}$ micro-wave ion source is also installed in this room, it is dedicated to ion implantation. Irradiation room 2 utilises medium-energy beams (from 3.4 to $10 \mathrm{MeV}$ for protons) from the tandem accelerator. Two beam lines are dedicated to an organism irradiation course with atmospheric micro-PIXE and middle-range ion implantation, respectively. Irradiation room 3 utilises a high-energy beam from the synchrotron. Vertical and horizontal (to be more accurate, 9.5 degrees falling download) beam lines are dedicated to medical research. A beam line in irradiation room 4 utilises highenergy beam from the synchrotron dedicates to radiation physics and biological research.

Installations of the accelerators and main beam lines were completed in December 1998. The beam commissioning was started at the beginning of 1999 . Injection into the synchrotron was first demonstrated on April 13, 1999. Beam tests to increase the extracted beam current were carried out using proton beams in July and September 1999, and March 2000. Extraction of $1.0 \times 10^{11}$ protons was demonstrated on March 17, 2000.

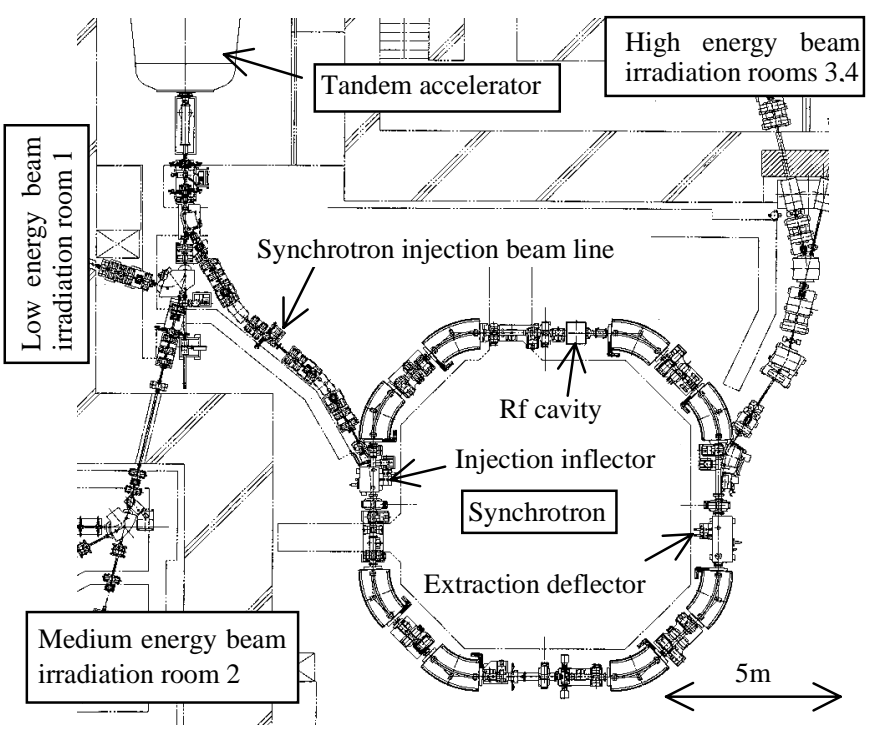

Figure 1: Configuration of W-MAST.

Performance of the synchrotron is summarised in this report.

\section{PERFORMANCE OF THE SYNCHROTRON}

The synchrotron accelerates protons and helium and carbon ions from the tandem accelerator. Maximum magnetic rigidity is $2.2 \mathrm{Tm}$, corresponds to $200 \mathrm{MeV}$ for protons and $55 \mathrm{MeV} /$ nucleon for fully stripped helium and carbon ions. Repetition rate of the operation is $2 \mathrm{~s}$. The ring consists of eight bending magnets, four long straight sections with focus type quadrupole magnets and four short straight sections with defocus type quadrupole magnets. The ring circumference is $33.2 \mathrm{~m}$. Average pressure in the vacuum chamber is about $4 \times 10^{-6} \mathrm{~Pa}$. Most beam studies were carried out using proton beams. Synchrotron performance for proton beams is discussed below.

\subsection{Injection}

$10 \mathrm{~mA}, 250 \mu$ s width pulsed $\mathrm{H}^{-}$beam is extracted from a negative ion source. The beam is focused and accelerated to $200 \mathrm{keV}$ by a Cockcroft type electrostatic preaccelerator. A kicker electrode chops the beam to a $0.5 \mathrm{~Hz}$, $20 \mu$ s width pulsed beam. The beam is injected into the tandem type electrostatic accelerator with a 5MV terminal voltage. In the canal at the $5 \mathrm{MV}$ terminal stage of the tandem accelerator, argon gas strips electrons from the $\mathrm{H}$ 
beam. Finally, the tandem accelerator ejects about a $6 \mathrm{~mA}$, $10 \mathrm{MeV}$ proton beam. A Schenckel type high-voltage power circuit realises acceleration of the pulsed, high peak current beam suitable for the synchrotron.

The proton beam from the tandem accelerator is injected into the synchrotron and accumulated through the horizontal multi-turn injection scheme using two injection bump magnets. Typically, the accumulated beam current measured by a current transducer is about $40 \mathrm{~mA}$, corresponds to $1.9 \times 10^{11}$ protons in the ring.

\subsection{Rf capture and acceleration}

An untuned accelerating cavity is installed in the synchrotron. The cavity employs Fe-based nanocrystalline FINEMET cores [3-4]. Since the untuned cavity has a small $\mathrm{Q}$ value, resonant frequency does not need to be controlled. In order to realise high voltage at the accelerating gap, impedance matching between the cavity and the feeding line from the rf amplifier is realised with the Multi-feed coupling technique [5].

Immediately after the injection, the proton beam is captured through an adiabatic rf capture process. The voltage of the rf cavity is increased from $0 \mathrm{~V}$ to $180 \mathrm{~V}$ lineally during this process. The injection beam has very low momentum dispersion, about $\pm 0.02 \%$. After the adiabatic rf capture, the voltage is increased to $400 \mathrm{~V}$ as the ramp rate. The beam is accelerated to $200 \mathrm{MeV}$ in $614 \mathrm{~ms}$. During the acceleration period, the frequency of the rf field supplying to the cavity is controlled according to the magnetic bending force measured by a search coil installed in one of the bending magnets in the ring. This search coil is printed on six plastic sheets and installed between the gap of this bending magnet and the vacuum chamber. This search coil measures the magnetic flux density including the effect of eddy current in the vacuum chamber.

In order to reduce the effect of space charge induced tune spread, the second order harmonic component was superposed onto the accelerating rf field. Since the untuned cavity has a small $Q$ value, the second order harmonic component is efficiently superposed together with the main accelerating rf field. Superposing the second order harmonic component during the capture period and initial stage of the acceleration reduces the peak charge density to about $70 \%$ and the accumulated protons after acceleration are increased to $150 \%$ [6]. Figure 2 shows an example of the numbers of circulating protons measured using a bunch monitor. The accelerating efficiency is about $55 \%$ and $1.1 \times 10^{11}$ protons are measured at the end of the acceleration.

\subsection{Tune Measurement}

Transverse tunes were measured by applying a transverse perturbation to the circulating beam. When frequency of the transverse perturbation is nearly equal to the betatron oscillation frequency, coherent betatron oscillation is excited. For the transverse perturbation, radio frequency field with wide band pseudo-random noise was applied using kicker plates (RFKs). RFKs consist of two pairs of parallel kicker plates. One kicker

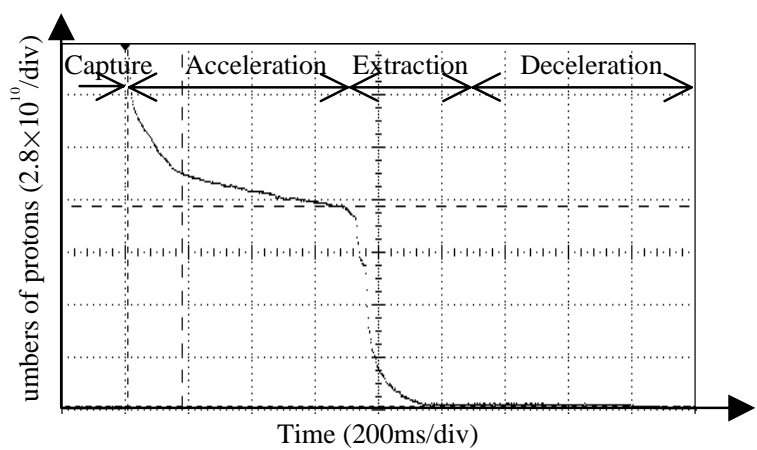

Figure 2: Numbers of circulating protons.

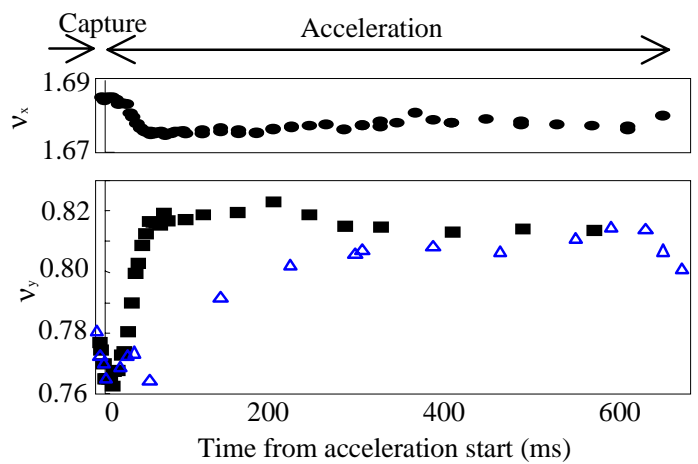

Figure 3: Measured horizontal and vertical tunes $\left(v_{x}\right.$, $\left.v_{y}\right)$. $=$ and $\Delta$ show the vertical tunes before and after the modification of the quadrupole magnets pattern, respectively.

for the horizontal plane, two vertical plates with $20 \mathrm{~cm}$ length along the beam are located in parallel at a separation distance of $14 \mathrm{~cm}$. The other kicker for the vertical plane, two horizontal plates with $8 \mathrm{~cm}$ length along the beam are located in parallel at a separation distance of $4 \mathrm{~cm}$.

The beam position monitor (BPM) output signal was analysed by a spectrum analyser. In observing this spectrum, peaks in the BPM response can be seen at $\mathrm{f}=\mathrm{nf}_{\mathrm{REV}}$ and $\mathrm{f}=(\mathrm{n} \pm \mathrm{v}) \mathrm{f}_{\mathrm{REV}}$ where $\mathrm{f}_{\mathrm{REV}}$ is the revolution frequency, $v$ is the mean betatron tune and $n$ is an integer. Measured tunes after rf capture are 1.69 and 0.80 for horizontal and vertical tunes, respectively.

Figure 3 shows the measured tunes. Defocusing force by the image charge of the beam in the vacuum chamber causes vertical tune falling during the capture period. The moving of tunes in the beginning of the accelerating period result from the sextupole field induced by the eddy current in the vacuum chamber between bending magnets. The tunes are corrected by modifying the pattern of the quadrupole magnets. This modification results in increasing the accumulated beam by $20 \%$.

\subsection{Extraction}

The horizontal third order resonance is utilised for a slow extraction scheme. Strength of all magnets is kept constant during the extraction [7]. Coherent betatron oscillation is excited using the transverse perturbation of the rf field with narrow band pseudo-random noise 
applied by the horizontal RFK, the same kicker as for horizontal tune measurements.

Conditions for the extraction are established using a period of $100 \mathrm{~ms}$ following the acceleration. The main quadrupole magnets are controlled to change the horizontal tune to 1.682. Two extraction bump magnets modify the horizontal closed orbit. The extraction bump orbit stretches the circumference of the closed orbit. As the revolution frequency has been kept constant since the end of the acceleration, the beam energy is slightly increased. Three sextupole magnets excite the horizontal third order resonance. Two of them, installed at symmetrical positions in the ring, are driven to excite the resonance. The third is driven to correct the chromaticity. Calculated separatrix size is $80 \pi \mathrm{mm} \cdot \mathrm{mrad}$. In order to smooth the extracted spill, the voltage of the accelerating rf cavity is decreased to $20 \mathrm{~V}$.

For the extraction, rf power is fed to the RFK. The spectrum of the rf field has a $20 \mathrm{kHz}$ band width covering the horizontal betatron oscillation frequency. Maximum voltage of the RFK is about $200 \mathrm{~V}$. Extraction efficiency is more than $90 \%$ and $1.0 \times 10^{11}$ protons are measured with a Faraday cup at the high-energy beam line.

Extracted beam current pattern is controlled using the gain pattern of the rf power amplifier. Figure 4 shows time structure of an extracted beam. In order to smooth the extracted spill, the gain of the RFK is controlled and about $50 \%$ of the accumulated protons are extracted. In the spectrum of the extracted beam, peaks derived from the $60 \mathrm{~Hz}$ commercial power line, at $720 \mathrm{~Hz}, 360 \mathrm{~Hz}$, and so on, are observed. This time structure is acceptable for the intended experiments, that is irradiation by a widely spread beam using wobbling magnets and scattering slabs.

The acceleration and extraction experiments were carried out for $200 \mathrm{MeV}, 180 \mathrm{MeV}, 160 \mathrm{MeV}, 140 \mathrm{MeV}$, $120 \mathrm{MeV}, 100 \mathrm{MeV}$ and $80 \mathrm{MeV}$. Transport to high-energy irradiation rooms was successfully demonstrated.

\subsection{Helium and carbon beams}

A $60 \mu \mathrm{A}$ continuous negative helium ion beam is realised using a bucket type positive helium ion source and a charge exchange cell with $\mathrm{Li}$ vapour. The beam is chopped by a kicker electrode and accelerated by the tandem accelerator. Then a $10 \mathrm{MeV}, 50 \mu \mathrm{A}$ pulsed $\mathrm{He}^{2+}$ beam with $50 \mu$ s width is injected into the synchrotron. $1.7 \times 10^{9}$ helium ions are accumulated after rf capture. About $80 \%$ of the beam is accelerated to $220 \mathrm{MeV}$ (55MeV/nucleon) and $90 \%$ of this amount is extracted under the same conditions as for the proton beam. $1.2 \times 10^{9}$ helium ions are measured with a Faraday cup at the highenergy beam line.

A $0.6 \mathrm{~mA}, 250 \mu$ s width pulsed negative carbon beam is extracted from the ion source, which is the same as for the proton beams, except for the target material. The beam is chopped by the kicker electrode and accelerated by the tandem accelerator. Then a $25 \mathrm{MeV}, 0.4 \mathrm{~mA}$ pulsed $\mathrm{C}^{4+}$ beam with $70 \mu$ s width is selected out using a bending magnet at downstream of the tandem accelerator. A carbon foil $\left(20 \mu \mathrm{g} / \mathrm{cm}^{2}\right)$ strips charge from the $\mathrm{C}^{4+}$ ions, $0.28 \mathrm{~mA} \mathrm{C}^{6+}$ is selected and injected into the synchrotron.

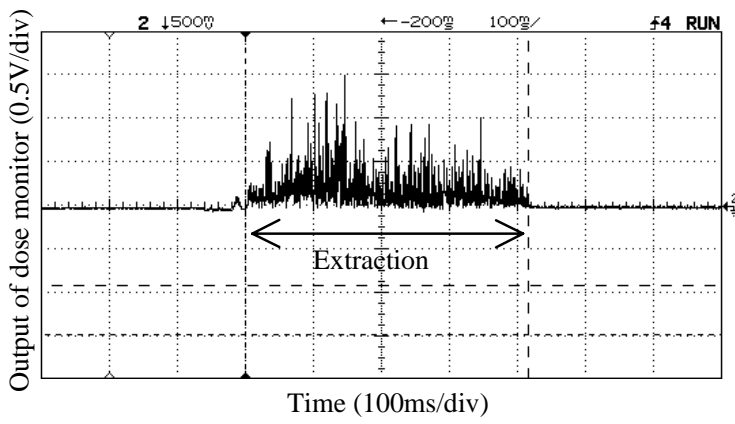

Figure 4: Time structure of extracted beam.

$2.8 \times 10^{9}$ carbon ions are accumulated after rf capture. About $30 \%$ of the beam is accelerated to $660 \mathrm{MeV}$ (55MeV/nucleon) and more than $90 \%$ of this amount is extracted under the same conditions as for the proton beam. A rather large emittance of the injection carbon beam and the beam scattering by residual gas in the vacuum chamber may cause the rather low acceleration efficiency. $8.3 \times 10^{8}$ carbon ions are measured with a Faraday cup at the high-energy beam line.

\section{CONCLUSION}

Commissioning of the synchrotron of W-MAST was successfully accomplished. $1.0 \times 10^{11}$ protons with $200 \mathrm{MeV}$ energy were extracted. $1.2 \times 10^{9}$ helium ions and $8.3 \times 10^{8}$ carbon ions with $55 \mathrm{MeV} /$ nucleon were also extracted under the same conditions as for the proton beam. The proton beam from the synchrotron has been utilised for some experiments. Additional beam tests for the irradiation systems are now being carried out.

\section{REFERENCES}

[1] S. Kakiuchi et al., "An Ion Synchrotron for Multipurposes Use of Energy Research Center, Wakasa Bay", Proc. of APAC98, p.790 (1998)

[2] Y. Ito et al., "Applications of Tandem/Synchrotron Accelerator to Physics, Engineering, Biotechnology and Proton Cancer Therapy", Proc. of APAC98, p.793 (1998)

[3] K. Saito, J. Hirota and F. Noda, "An Untuned RF Cavity Loaded with Fe-based Nanocrystalline FINEMET Cores", Beam Science and Technology, Kyoto Univ. 2, p.15 (1997)

[4] K. Saito et al., "FINEMET-core Loaded Untuned RF Cavity", Nucl. Instr. and Meth. A402, p.1 (1998)

[5] Y. Iwashita. "Ferro-magnetic Material Loaded Untuned RF Cavity for Synchrotron", Jpn. J. Appl Phys. 36, L727 (1997)

[6] K. Saito et al., "RF Accelerating System for A Compact Ion Synchrotron", in this conference.

[7] K. Hiramoto et al., Nucl. Instr. and Meth. A322, p.154 (1992) 